\title{
Induced Casimir Force between Heavy Particles Substituted in an Oscillator Chain
}

\author{
F. Chegini*, F. Kheirandish and M.R. Setare \\ Department of Physics, Faculty of Science, University of Kurdistan, P.O. Box 66177-15175, Sanandaj, Iran \\ (Received December 5, 2018; revised version March 27, 2019; in final form March 31, 2019)

\begin{abstract}
The induced Casimir energy for a subsystem consisting of two heavy particles substituted at a finite distance in a linear chain of interacting oscillators is obtained. The heavy particles are linearly interacting with the nearest neighbor particles. A continuum modeling for the discrete case is considered and the corresponding Casimir energy is calculated. The result obtained from the continuum modeling agrees with the discrete model qualitatively.
\end{abstract}

DOI: 10.12693/APhysPolA.136.66

PACS/topics: 42.50.Lc, 05.40.Jc, 03.70. $+\mathrm{k}$

\section{Introduction}

The Casimir effect, originally introduced by Casimir in 1948 [1], can be considered as a macroscopic quantum effect originating from quantum vacuum fluctuations in the presence of some boundary conditions and plays an important role in various fields of physics such as the quantum field theory, condensed matter physics, cosmology, atomic and molecular physics, and also nanotechnology [2-7]. The Casimir energy is the energy difference of zero-point energy of a quantum field in the presence and absence of some external fields or boundary conditions. This energy is a measurable quantity depending on various factors such as configuration or geometry of the problem, temperature, and the material used.

Impurities substituted in one-dimensional lattice can be modeled by a linear chain of interacting harmonic oscillators with nearest-neighbors interactions. The impurities are distinguished oscillators which can be considered as a subsystem interacting with the rest of the chain as its reservoir. The time evolution of the autocorrelation and cross-correlation functions related to momenta and displacements of these impurities are studied in [8]. The vibrational self-energy defined as the difference between the zero-point energy of the lattice in the presence of defects and without them has been calculated in $[9,10]$. The energy spectrum of these lattices has been investigated in [11].

For investigating the quantum dynamics of a real physical system, the effect of its environment is unavoidable on different physical quantities of the subsystem. The environment of a physical system can be modeled by different methods and its effects may be taken into account phenomenologically inspired by experimental results. The investigation of such systems falls into the domain of open quantum systems which has been dealt with extensively $[12,13]$. One of the most successful models of a reservoir or environment is the Caldeira-Leggett

*corresponding author; e-mail: chegini.sepideh@gmail.com model [14-17]. In this model the environment of a system is modeled by a collection of independent harmonic oscillators with different frequencies. The system of interest couples linearly to the environment oscillators via some coupling coefficients which are related to the susceptibility or response function of the environment. This model has been applied to a variety of different problems in atomic and molecular physics and condensed matter, where dissipation on energy or phase are important or even when the medium is an amplifying one. Some important examples are: quantum Brownian motion of an oscillator in a dissipative medium [18], quantum tunneling with dissipation $[8,15]$, electromagnetic field quantization in a magnetodielectric medium [19-21], Casimir energy for separated objects in vacuum electromagnetic field [1, 22-24], dissipative optomechanical models [25], and dissipation in quantum optics [26].

In the present work, we first consider the quantum Brownian motion of an oscillator linearly interacting with a reservoir and find the Casimir energy of the system by diagonalizing the Hamiltonian. Then, we consider the Casimir energy induced between two distinguished oscillators substituted in a linear chain. Following the method introduced in [27], we will consider a field model for this case as a continuum limit of the chain in the presence of two delta-potentials located at a finite distance and find the Casimir energy induced between these impurities due to the field fluctuations of the continuum chain. The result obtained from the continuum modeling is in agreement with the discrete case qualitatively.

\section{An oscillator interacting with a heat bath}

This model is the prototype of some important quantum dissipative models like quantum Brownian motion [14] or electromagnetic field quantization in a linear dispersive and absorptive magnetodielectric medium [1921]. In this model, the main system, considered here as a harmonic oscillator, is coupled linearly to a collection of harmonic oscillators modeling its environment, Fig. 1. The Hamiltonian of the total system is 


$$
\begin{aligned}
H & =\frac{1}{2}\left(P^{2}+\Omega_{0}^{2} Q^{2}\right)+\frac{1}{2} \sum_{n=1}^{N}\left(p_{n}^{2}+\omega_{n}^{2} q_{n}^{2}\right) \\
& +\frac{1}{2} \sum_{n=1}^{N} \epsilon_{n}\left(q_{n} Q+Q q_{n}\right),
\end{aligned}
$$

where $Q$ and $q_{n}$ represent the coordinates, $P$ and $p_{n}$ the canonical conjugate momenta of the system and the environment, respectively. The main oscillator has frequency $\Omega_{0}$ and $\omega_{n}$ 's are the frequencies of the oscillators in the bath. The coupling constants are denoted by $\epsilon_{n}$. Since the Hamiltonian in Eq. (1) is quadratic in both coordinates and momenta, the equations of motion can be solved exactly [8]. Under the canonical transformations

$$
\begin{aligned}
& P=\sum_{\nu=0}^{N} X_{0 \nu} p_{\nu}^{\prime}, \quad Q=\sum_{\nu} X_{0 \nu} q_{\nu}^{\prime}, \\
& p_{n}=\sum_{\nu} X_{n \nu} p_{\nu}^{\prime}, \quad q_{n}=\sum_{\nu} X_{n \nu} q_{\nu}^{\prime},
\end{aligned}
$$

Hamiltonian is diagonalized in normal modes [8]:

$$
H=\frac{1}{2} \sum_{\nu}\left(p_{\nu}^{\prime 2}+s_{\nu}^{2} q_{\nu}^{\prime 2}\right)
$$

where $s_{\nu}$ are eigenvalues of equation

$$
V X_{\nu}=z_{\nu} X_{\nu}
$$

and $s_{\nu}^{2}=z_{\nu}$. The matrix $V$ is the interaction matrix

$$
V=\left(\begin{array}{cccc}
\Omega_{0}^{2} & \epsilon_{1} & \cdots & \epsilon_{n} \\
\epsilon_{1} & \omega_{1}^{2} & & \\
\vdots & & \ddots & \\
\epsilon_{n} & & & \omega_{n}^{2}
\end{array}\right)
$$

and $X_{\nu}=\left(X_{0 \nu}, X_{1 \nu}, \cdots, X_{n \nu}\right)$. The squared frequencies satisfy the secular equation

$$
G(z)=\Omega_{0}^{2}-z-\sum_{n} \epsilon_{n}^{2}\left(\omega_{n}^{2}-z\right)^{-1}=0 .
$$

Now from Hamiltonian (3), the zero-point energy of this system is

$$
F=\sum_{\nu=0}^{N} \frac{\hbar}{2} s_{\nu}
$$

From the argument theorem, we have

$$
\frac{1}{2 \pi i} \oint_{C} x \frac{f^{\prime}(x)}{f(x)} \mathrm{d} x=\left[\sum x_{i}\right]_{f\left(x_{i}\right)=0}-\left[\sum x_{i}\right]_{f\left(x_{i}\right)=\infty},
$$

where the summations are over the zeros and the poles of the function $f$ inside the contour of integration, respectively. Contour integral $C$ is a closed and counterclockwise path in the complex plane which consists of a straight line parallel to the imaginary axis closed by a semicircle at infinity in the right half plane. So we have [28]:

$$
F=\sum_{n=1}^{N} \frac{\hbar}{2} \omega_{n}+\frac{\hbar}{4 \pi \mathrm{i}} \oint_{C} s \frac{\mathrm{d}}{\mathrm{d} s} \ln G(s) \mathrm{d} s .
$$

Integrating along the imaginary axis from $\infty$ to $-\infty$ and integrating by parts and also introducing a new variable $s=i \xi$ (the Wick rotation), we find

$$
\begin{aligned}
F & =\sum_{n=1}^{N} \frac{\hbar}{2} \omega_{n} \\
& +\frac{\hbar}{4 \pi} \int_{-\infty}^{\infty} \ln \left(\Omega_{0}^{2}+\xi^{2}-\sum_{n=1}^{N} \epsilon_{n}^{2}\left(\omega_{n}^{2}+\xi^{2}\right)^{-1}\right) \mathrm{d} \xi
\end{aligned}
$$

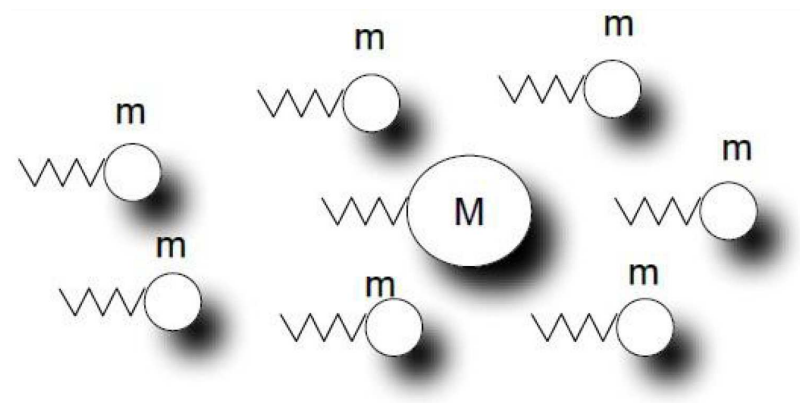

Fig. 1. The main oscillator interacts linearly with bath oscillators.

\section{Two heavy particles interacting with a linear chain}

Let us consider two distinguished heavy particles or impurities substituted in a linear chain [8-10]. In this model the heavy particles are linearly coupled to their nearest neighbours, as shown in Fig. 2. In the equilibrium state, the distance between atoms is the lattice constant $a_{0}$ and positions of atoms are considered as deviations around their equilibrium position. The Hamiltonian of the total system is [8]

$$
\begin{aligned}
H & =\frac{P_{+}^{\prime 2}}{2 M_{+}}+\frac{P_{-}^{\prime 2}}{2 M_{-}}+\sum_{l=1}^{L} \frac{p_{l}^{\prime 2}}{2 m}+\sum_{n=1}^{N} \frac{p_{n}^{\prime 2}}{2 m}+\sum_{k=1}^{K} \frac{p_{k}^{\prime 2}}{2 m} \\
& +\frac{1}{2} g^{\prime}\left[\sum_{l=1}^{L-1}\left(q_{l+1}^{\prime}-q_{l}^{\prime}\right)^{2}+\sum_{k=1}^{K-1}\left(q_{k+1}^{\prime}-q_{k}^{\prime}\right)^{2}\right. \\
& \left.+\sum_{n=1}^{N-1}\left(q_{n+1}^{\prime}-q_{n}^{\prime}\right)^{2}\right]+\frac{1}{2} g^{\prime}\left[\left(q_{k=1}^{\prime}-Q_{-}^{\prime}\right)^{2}\right. \\
& \left.+\left(Q_{-}^{\prime}-q_{l=1}^{\prime}\right)^{2}+\left(q_{l=L}^{\prime}-Q_{+}^{\prime}\right)^{2}+\left(Q_{+}^{\prime}-q_{n=1}^{\prime}\right)^{2}\right],
\end{aligned}
$$

where $g^{\prime}$ is a coupling constant and $m, q_{j}^{\prime}$ and $p_{j}^{\prime}$ are mass, position, and momentum of the chain oscillators, respectively. The mass of heavy particles are denoted by $M_{ \pm}$. Also $Q_{ \pm}^{\prime}$ and $P_{ \pm}^{\prime}$ denote coordinates and momenta of heavy particles, respectively. There are $L$ particles between the heavy particles and on the outer sides of the chain, there are $K$ and $N$ particles of the chain.

For convenience we redefine the variables

$$
\begin{aligned}
& M^{1 / 2} P_{ \pm}=P_{ \pm}^{\prime}, \quad M^{-1 / 2} Q_{ \pm}=Q_{ \pm}^{\prime}, \\
& m^{1 / 2} p_{j}=p_{j}^{\prime}, \quad m^{-1 / 2} q_{j}=q_{j}^{\prime}, \quad g=g^{\prime} / m,
\end{aligned}
$$




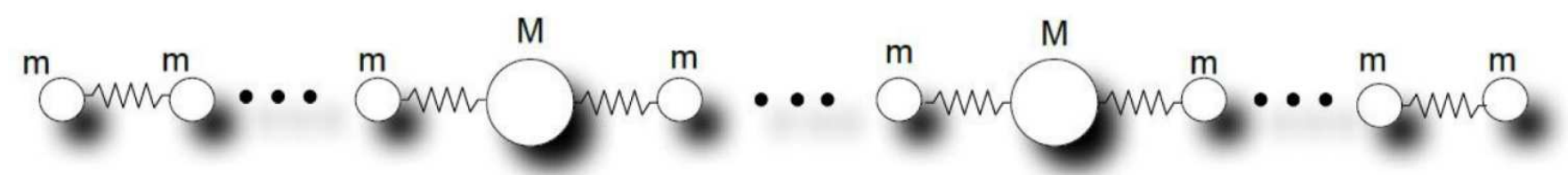

Fig. 2. Two heavy particles substituted in a linear chain with nearest-neighbors couplings.

$$
\begin{aligned}
& p_{k}=\sum_{e=1}^{K} C_{k e} p_{e}^{\prime}, \quad q_{k}=\sum_{e=1}^{K} C_{k e} q_{e}^{\prime}, \\
& p_{n}=\sum_{a=1}^{N} C_{n a} p_{a}^{\prime}, \quad q_{n}=\sum_{a=1}^{N} C_{n a} q_{a}^{\prime}, \\
& p_{l}=\sum_{b=1}^{L} C_{l b} p_{b}^{\prime}, \quad q_{l}=\sum_{b=1}^{L} C_{l b} q_{b}^{\prime},
\end{aligned}
$$

where

$$
\begin{aligned}
& C_{k e}=\frac{2}{\sqrt{2 K+1}} \sin \left(k \frac{2 e-1}{2 K+1} \pi\right), \quad e=1, \cdots, K \\
& C_{n a}=\frac{2}{\sqrt{2 N+1}} \sin \left(n \frac{2 a-1}{2 N+1} \pi\right), \quad a=1, \cdots, N \\
& C_{l b}=\frac{2}{\sqrt{L+1}} \sin \left(l \frac{b}{L+1} \pi\right), \quad b=1, \cdots, L
\end{aligned}
$$

The total Hamiltonian in redefined dynamical variables is

$$
\begin{gathered}
H_{\text {total }}=\frac{P_{ \pm}^{2}}{2}+\frac{1}{2} \Omega_{ \pm}^{2} Q_{ \pm}^{2}+\sum_{e=1}^{K} \frac{1}{2}\left(p_{e}^{2}+\omega_{e}^{2} q_{e}^{2}\right) \\
+\sum_{a=1}^{N} \frac{1}{2}\left(p_{a}^{2}+\omega_{a}^{2} q_{a}^{2}\right)+\sum_{b=1}^{L} \frac{1}{2}\left(p_{b}^{2}+\omega_{b}^{2} q_{b}^{2}\right) \\
+Q_{+}\left(\sum_{b} \epsilon_{b}^{+} q_{b}+\sum_{a} \epsilon_{a} q_{a}\right) \\
+Q_{-}\left(\sum_{b} \epsilon_{b}^{-} q_{b}+\sum_{e} \epsilon_{e} q_{e}\right),
\end{gathered}
$$

where

$$
\begin{aligned}
& \Omega_{ \pm}^{2}=2 g^{\prime} / M_{ \pm}, \quad \omega_{a}^{2}=4 g \sin ^{2}\left(\frac{2 a-1}{2(2 N+1)} \pi\right) \\
& \omega_{b}^{2}=4 g \sin ^{2}\left(\frac{b}{2(L+1)} \pi\right) \\
& \omega_{e}^{2}=4 g \sin ^{2}\left(\frac{2 e-1}{2(2 K+1)} \pi\right) \\
& \epsilon_{a}=-2 g \sqrt{\left(\frac{m / M_{+}}{2 N+1}\right)} \sin \left(\frac{2 a-1}{2 N+1} \pi\right) \\
& \epsilon_{e}=-2 g \sqrt{\left(\frac{m / M_{-}}{2 K+1}\right)} \sin \left(\frac{2 e-1}{2 K+1} \pi\right)
\end{aligned}
$$

$$
\begin{aligned}
& \epsilon_{b}^{+}=-g \sqrt{\left(\frac{2 m / M_{+}}{L+1}\right)} \sin \left(\frac{b}{L+1} L \pi\right), \\
& \epsilon_{b}^{-}=-g \sqrt{\left(\frac{2 m / M_{-}}{L+1}\right)} \sin \left(\frac{b}{L+1} \pi\right) .
\end{aligned}
$$

By making use of the following canonical transformations:

$$
\begin{aligned}
& P_{ \pm}=\sum_{\alpha} X_{ \pm \alpha} p_{\alpha}, \quad Q_{ \pm}=\sum_{\alpha} X_{ \pm \alpha} q_{\alpha} \\
& p_{i}=\sum_{\alpha} X_{i \alpha} p_{\alpha}, \quad q_{i}=\sum_{\alpha} X_{i \alpha} q_{\alpha}, \quad i=a, b, e,
\end{aligned}
$$

the Hamiltonian (15) transforms to a quadratic form

$$
\begin{aligned}
& H=\frac{1}{2} \sum_{\alpha}\left(p_{\alpha}^{2}+s_{\alpha}^{2} q_{\alpha}^{2}\right), \\
& \alpha=1, \cdots, N+K+L+2,
\end{aligned}
$$

describing a system consisting of $N+K+L+2$ independent harmonic oscillators. The eigenvalue equation can be obtained similar to the previous section as

$$
V X_{\alpha}=z_{\alpha} X_{\alpha}
$$

where $V$ is the interaction matrix

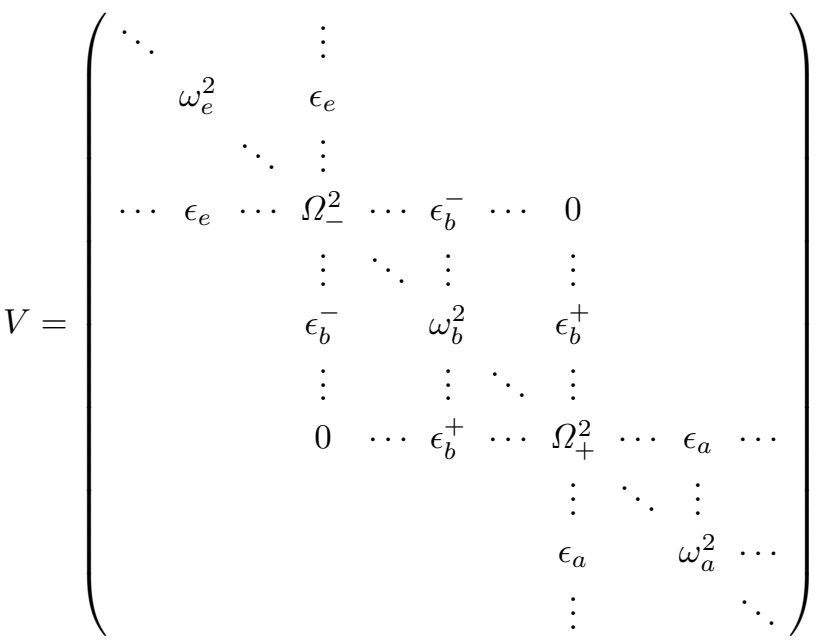

and $X_{\alpha}=\left(\cdots, X_{e \alpha}, \cdots, X_{-\alpha}, \cdots, X_{b \alpha}, \cdots, X_{+\alpha}, \cdots\right.$, $\left.X_{a \alpha}, \cdots\right)$. For convenience, we set $K=N$ and $M_{+}=$ $M_{-}$, and the secular equation is obtained as

$$
G_{+} G_{-}=0 \text {. }
$$

The normal frequencies $s_{\alpha}^{2}=z_{\alpha}$ are the roots of Eq. (21) where

$$
G_{ \pm}=s^{2}-\Omega^{2}-F(s)-H_{+}(s) \mp H_{-}(s),
$$




$$
\begin{aligned}
& F(s)=\sum_{a=1}^{N} \frac{\epsilon_{a}^{2}}{s^{2}-\omega_{a}^{2}}, \quad H_{+}(s)=\sum_{b=1}^{L} \frac{\epsilon_{b}^{+2}}{s^{2}-\omega_{b}^{2}}, \\
& H_{-}(s)=\sum_{b=1}^{L} \frac{\epsilon_{b}^{+} \epsilon_{b}^{-}}{s^{2}-\omega_{b}^{2}} .
\end{aligned}
$$

Following the usual method to find the Casimir energy between parallel neutral plates, here we find the difference of the zero-point energy of the system for two different configurations. In the first configuration the substituted massive impurities are separated by finite $L \ll N$ and in the second configuration they are separated at maximum distance, that is $L=N$ [8]. By making use of the argument principle, we have

$$
\begin{aligned}
E & =\Delta E_{c}=\sum_{\alpha} \frac{\hbar}{2} s_{\alpha}-\sum_{\alpha} \frac{\hbar}{2} s_{\alpha}^{\prime}= \\
& \frac{\hbar \omega_{0}}{2 \pi} \int_{0}^{\infty} \ln \left(G_{+} G_{-} / G_{+}^{\prime} G_{-}^{\prime}\right) \mathrm{d} \xi \\
& +\frac{\hbar}{2} \sum_{a=1}^{N} \omega_{a}-\frac{\hbar}{2} \sum_{a=1}^{N} \omega_{a}^{\prime}+\frac{\hbar}{2} \sum_{b=1}^{L} \omega_{b}-\frac{\hbar}{2} \sum_{b=1}^{L} \omega_{b}^{\prime},
\end{aligned}
$$

where $\omega_{0}=\sqrt{4 g}=2 \sqrt{g^{\prime} / m}$ is an upper bound for normal mode frequencies given in Eq. (16), and

$$
\begin{aligned}
G_{ \pm} & =\xi^{2}+m /(2 M)-\sum_{a=1}^{N} \frac{\frac{m / 4 M}{2 N+1} \sin ^{2}\left(\frac{2 a-1}{2 N+1} \pi\right)}{\xi^{2}+\sin ^{2}\left(\frac{2 a-1}{2(2 N+1)} \pi\right)} \\
\mp & \sum_{b=1}^{L} \frac{\frac{m / 8 M}{L+1} \sin \left(\frac{b}{L+1} \pi\right) \sin \left(\frac{b}{L+1} L \pi\right)}{\xi^{2}+\sin ^{2}\left(\frac{b}{2(L+1)} \pi\right)} \\
& -\sum_{b=1}^{L} \frac{\frac{m / 8 M}{L+1} \sin ^{2}\left(\frac{b}{L+1} L \pi\right)}{\xi^{2}+\sin ^{2}\left(\frac{b}{2(L+1)} \pi\right)} .
\end{aligned}
$$

Prime denotes the case where the distance between heavy particles is taken to be large $(N=L=500)$. Figure 3 shows the behavior of the energy difference as a function

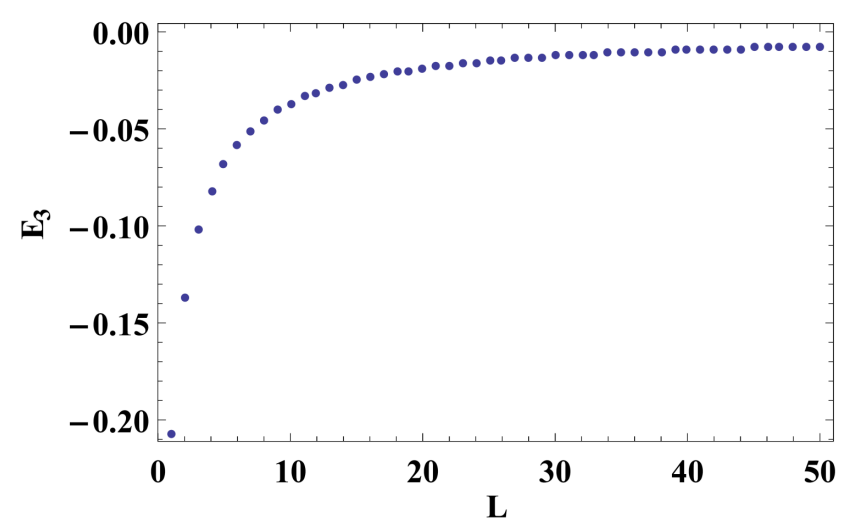

Fig. 3. The behavior of normalised energy $E_{3}=$ $E /\left(\hbar \omega_{0} / 2 \pi\right)$, as a function of distance between impurities $L$ (in unit of lattice constant $a_{0}$ ) for $m / M=10^{-6}$. of $L$ for $m / M=10^{-6}$. It is seen that an attractive force $F=-\frac{\partial E}{\partial L}$ is induced between the massive impurities due to quantum fluctuations of the quantum chain.

\section{Continuum limit}

In this section, we consider the continuum case of the discrete oscillator model. In this case, oscillator displacement is replaced with a scalar field $u(x, t)$. The heavy particles are assumed to be located at positions $x=0$ and $x=a$. The field $u(x, t)$ satisfies the Dirichlet boundary conditions $(u(0, t)=u(a, t)=0)$ in the place of heavy particles. Following [27], the Dirichlet boundary conditions can be modeled by a linear combination of Dirac delta-potentials

$$
V(x)=\lambda \delta(x)+\lambda \delta(x-a)
$$

where $\lambda$ determines the strength of interaction which in the case of very heavy defects tends to infinity and accordingly this imposes Dirichlet boundary conditions $u(0, t)=u(a, t)=0$ on the displacement field. The field $u(x, t)$ satisfies [29]:

$$
\left(\frac{1}{v^{2}} \frac{\partial^{2}}{\partial t^{2}}-\frac{\partial^{2}}{\partial x^{2}}+V(x)\right) u(x, t)=0,
$$

where $v$ is the velocity of propagation. Note that in the continuum model the lattice distance $a_{0}$ and the particle mass $m$ tend to zero but the coupling constant (spring constant) $g^{\prime}$ defined in (11) and $g=g^{\prime} / m$ tend to infinity such that the velocity of propagation defined by [29]:

$$
v=\lim _{\substack{a_{0} \rightarrow 0 \\ g^{\prime} / m \rightarrow \infty}} \sqrt{a_{0}^{2} \frac{g^{\prime}}{m}}=\lim _{\substack{a_{0} \rightarrow 0 \\ g \rightarrow \infty}} a_{0} \sqrt{g},
$$

remains constant. Therefore, a connection between $v, a_{0}$ and $\omega_{0}$ can be established approximately as $v \approx a_{0} \sqrt{g}=$ $a_{0} \omega_{0} / 2$ for sufficiently small lattice distances. Therefore, by considering a very large number of atoms and a very small lattice constant the discrete model can arbitrarily approach the continuum model and the difference between energies in discrete and continuum model will decrease by increasing the number of atoms and decreasing the lattice constant.

The Green function of (27) fulfills

$$
\begin{aligned}
& \left(\frac{1}{v^{2}} \frac{\partial^{2}}{\partial t^{2}}-\frac{\partial^{2}}{\partial x^{2}}+V(x)\right) G\left(x, t ; x^{\prime}, t^{\prime}\right)= \\
& \quad \delta\left(x-x^{\prime}\right) \delta\left(t-t^{\prime}\right),
\end{aligned}
$$

and using the Fourier transform, the Green function can be expanded in frequency space components as

$$
G\left(x, t ; x^{\prime}, t^{\prime}\right)=\int_{-\infty}^{\infty} \frac{\mathrm{d} \omega}{2 \pi} \mathrm{e}^{-\mathrm{i} \omega\left(t-t^{\prime}\right)} g\left(x, x^{\prime} ; \omega\right) .
$$

By inserting (30) into (29), we find

$$
\begin{aligned}
& \left(-\frac{\partial^{2}}{\partial x^{2}}-k^{2}+\lambda \delta(x)+\lambda \delta(x-a)\right) g\left(x, x^{\prime} ; \omega\right)= \\
& \quad \delta\left(x-x^{\prime}\right),
\end{aligned}
$$


where $k^{2}=\omega^{2} / v^{2}$ and the solution is given by

$$
\begin{aligned}
& g\left(x, x^{\prime} ; \omega\right)=g_{0}\left(x, x^{\prime} ; \omega\right)-\lambda g_{0}(x, 0 ; \omega) \frac{\left[1+\lambda g_{0}(a, a ; \omega)\right] g_{0}\left(0, x^{\prime} ; \omega\right)-\lambda g_{0}(0, a ; \omega) g_{0}\left(a, x^{\prime} ; \omega\right)}{\Delta} \\
& -\lambda g_{0}(x, a ; \omega) \frac{\left[1+\lambda g_{0}(0,0 ; \omega)\right] g_{0}\left(a, x^{\prime} ; \omega\right)-\lambda g_{0}(a, 0 ; \omega) g_{0}\left(0, x^{\prime} ; \omega\right)}{\Delta},
\end{aligned}
$$

where

$$
g_{0}\left(x, x^{\prime} ; \omega\right)=\frac{\mathrm{i}}{2|\omega|} \mathrm{e}^{\mathrm{i}|\omega|\left|x-x^{\prime}\right|},
$$

is the Green function in the absence of impurities and $\Delta=1+\lambda^{2}\left[g_{0}(0,0 ; \omega) g_{0}\left(a, a^{\prime} ; \omega\right)-g_{0}(0, a ; \omega) g_{0}(a, 0 ; \omega)\right]$

$$
+\lambda\left[g_{0}(0,0 ; \omega)+g_{0}(a, a ; \omega)\right] .
$$

The Green function is related to the correlation of displacement field as

$$
G\left(x, t ; x^{\prime}, t^{\prime}\right)=\mathrm{i}\left\langle\hat{T} u(x, t) u\left(x^{\prime}, t^{\prime}\right)\right\rangle,
$$

where $\hat{T}$ is the time-ordering operator. The energy density can be determined from 00 component of energymomentum tensor given by

$$
T^{\mu \nu}=\partial^{\mu} \varphi \partial^{\nu} \varphi-\frac{1}{2} g^{\mu \nu} \partial_{\eta} \varphi \partial^{\eta} \varphi
$$

and therefore,

$$
\begin{aligned}
& E=\int \mathrm{d} x\left\langle T^{00}\right\rangle \\
& =\left.\int \frac{\mathrm{d} \omega}{2 \pi} \frac{1}{2 i}\left(\frac{\omega^{2}}{v^{2}}+\frac{\partial}{\partial x} \frac{\partial}{\partial x^{\prime}}\right) g\left(x, x^{\prime} ; \omega\right)\right|_{x=x^{\prime}} .
\end{aligned}
$$

By inserting (32) into (37) and considering the limit $\lambda \rightarrow \infty$ (very massive defects), one finds

$$
E=-\frac{\hbar v \pi}{24 a}, \rightarrow \frac{E}{\left(\hbar v / a_{0}\right)}=-\frac{\pi / 24}{\left(a / a_{0}\right)},
$$

in agreement with [27]. Therefore, the induced force between the defects is

$$
F=-\frac{\partial E(a)}{\partial a}=-\frac{\hbar v \pi}{24 a^{2}} .
$$

In Figs. 3 and 4 , the behavior of dimensionless energies $E /\left(\hbar \omega_{0} / 2 \pi\right)$ and $E /\left(2 \hbar v / a_{0}\right)$ in terms of the distance $L$ (in unit of lattice constant $a_{0}$ ) are depicted, respectively. The diagrams qualitatively have the same behaviour.

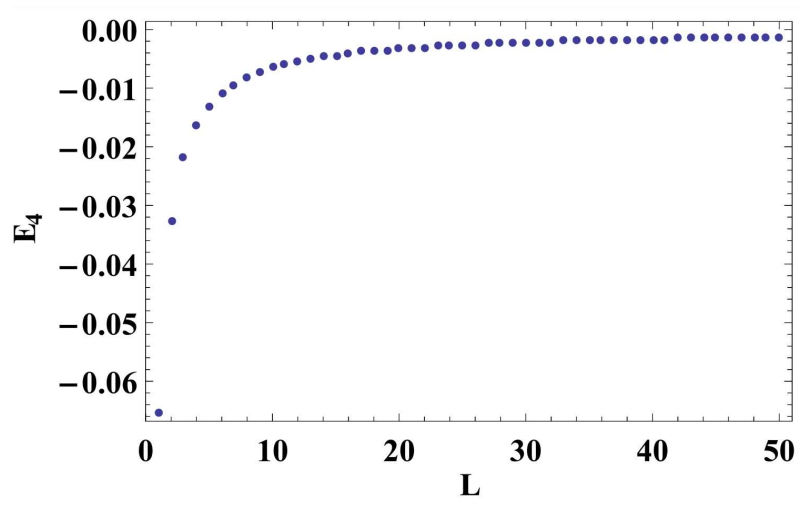

Fig. 4. The behavior of dimensionless energy $E_{4}=$ $E /\left(2 \hbar v / a_{0}\right)$ in the continuum model in $L$.
The ratio of the scaled energy in discrete model $\left(E_{3}\right)$ to the scaled energy in continuum model $\left(E_{4}\right)$ is depicted in Fig. 5 in terms of $L$. For large values of $L$ this ratio is approximately constant $\left(E_{3} / E_{4} \approx 5.66\right)$ and using $v \approx$ $a_{0} \omega_{0} / 2$, we find

$$
\frac{E_{3}}{E_{4}}=\frac{4 \pi v}{a_{0} \omega_{0}} \approx 2 \pi=6.28
$$

with a relative error $\approx 11 \%$.

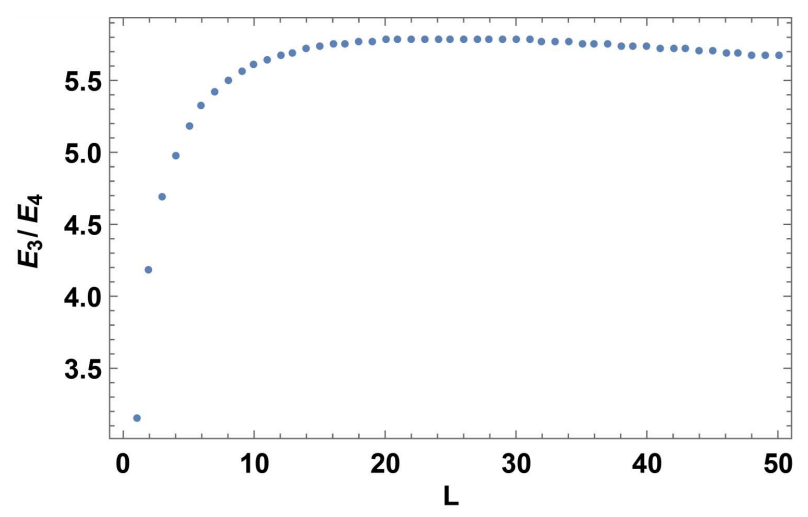

Fig. 5. The ratio of scaled energies depicted in Figs. 3 and 4. For large values of $L(L \approx 50)$ this ratio is approximately 5.66 for number of atoms $N=500$.

\section{Conclusion}

By diagonalizing the Hamiltonian and using the argument principle, the induced Casimir energy for a subsystem consisting of two heavy particles substituted at a finite distance in a linear chain of interacting oscillators was obtained. The heavy particles were linearly interacting with the nearest neighbor particles. The continuum model of the oscillator chain was considered and the corresponding Casimir energy was reobtained based on the method introduced in [27]. The result obtained from the continuum modeling was in agreement with the discrete model qualitatively.

\section{References}

[1] H.B.G. Casimir, Proc. K. Ned. Akad. Wet. 51, 793 (1948).

[2] M. Bordag, U. Mohideen, V.M. Mostepanenko, Phys. Rep. 353, 1 (2001).

[3] M. Bordag, G.L. Klimchitskaya, U. Mohideen, V.M. Mostepanenko, Advances in the Casimir Effect, Oxford University Press, Oxford 2008. 
[4] E.V. Blagov, G.L. Klimchitskaya, V.M. Mostepanenko, Phys. Rev. B 75, 235413 (2007); E.V. Blagov, G.L. Klimchitskaya, V.M. Mostepanenko, Phys. Rev. $B$ 71, 235401 (2005).

[5] E. Buks, M.L. Roukes, Phys. Rev. B 63, 033402 (2001).

[6] K.A. Milton, The Casimir Effect: Physical Manifestations of Zero-Point Energy, World Sci., Singapore 2001.

[7] E. Elizalde, S.D. Odintsov, A. Romeo, A.A. Bytsenko, S. Zerbini, Zeta Regularization Techniques with Applications, World Sci., 1994.

[8] P. Ullersma, Physica 32, 27 (1966); P. Ullersma, J.A. Tjon, Physica 71, 294 (1974).

[9] E.W. Montroll, R.B. Potts, Phys. Rev. 100, 525 (1955).

[10] J. Hori, T. Asahi, Prog. Theor. Phys. 17, 523 (1957).

[11] S. Takeno, Prog. Theor. Phys. 28, 1 (1962).

[12] H.P. Breuer, F. Petruccione, Theory of Open Quantum Systems, Oxford University Press, Oxford 2002.

[13] U. Weiss, Quantum Dissipative Systems, World Sci., Singapore 1999.

[14] A.O. Caldeira, An Introduction to Macroscopic Quantum Phenomena and Quantum Dissipation, Cambridge University Press, 2014.

[15] A.O. Caldeira, A.J. Leggett, Phys. Rev. Lett. 46, 211 (1981).
[16] A.O. Caldeira, A.J. Leggett, Ann. Phys. 149, 374 (1983).

[17] A.J. Leggett, Phys. Rev. B 30, 1208 (1984).

[18] E. Amooghorban, F. Kheirandish, Int. J. Theor. Phys. 53, 2593 (2014).

[19] F. Kheirandish, M. Amooshahi, Phys. Rev. A 74, 042102 (2006); ibid., 76, 062103 (2007)10. 1103/PhysRevA.76.06210.

[20] F. Kheirandish, M. Amooshahi, M. Soltani, J. Phys. B Mol. Opt. Phys. 42, 075504 (2009).

[21] F. Kheirandish, M. Soltani, Phys. Rev. A 78, 012102 (2008).

[22] F. Kheirandish, E. Amooghorban, M. Soltani, Phys. Rev. A 83, 032507 (2011).

[23] F. Kheirandish, M. Jafari, Phys. Rev. A 86, 022503 (2012)

[24] F. Kheirandish, S. Salimi, Phys. Rev. A 84, 062122 (2011).

[25] W.P. Bowen, G.J. Milburn, Quantum Optomechanics, Taylor and Francis, Florida 2016.

[26] W. Schleich, Quantum Optics in Phase Space, WileyVCH, Berlin 2001.

[27] K.A. Milton, J. Phys. A 37, 6391 (2004).

[28] S.K. Lamoreaux, Rep. Prog. Phys. 68, (2005).

[29] W. Greiner, J. Reinhardt, Field Quantization, Springer-Verlag, Berlin 1996. 